\title{
A Mechanically Tunable Plasmonic Structure Composed of a Monolayer Array of Metal-Capped Colloidal Spheres on an Elastomeric Substrate
}

\author{
Xiaolong Zhu', Lei Shi ${ }^{1}$, Xiaohan Liu ${ }^{1}(\varangle)$, Jian Zi ${ }^{1}(\bowtie)$, and Zhenlin Wang ${ }^{2}$ \\ ${ }^{1}$ Department of Physics, Key Laboratory of Micro and Nano Photonic Structures (Ministry of Education), and State Key Laboratory of \\ Surface Physics, Fudan University, Shanghai 200433, China \\ ${ }^{2}$ National Laboratory of Solid State Microstructures, Nanjing University, Nanjing 210093, China \\ Received: 31 July 2010 / Revised: 15 September 2010 / Accepted: 16 September 2010 \\ C The Author(s) 2010. This article is published with open access at Springerlink.com
}

\begin{abstract}
A highly stretchable plasmonic structure composed of a monolayer array of metal-capped colloidal spheres on an elastomeric substrate has been fabricated using simple and inexpensive self-assembly and transfer-printing techniques. This composite structure supports coupled surface plasmons whose wavelengths are sensitive to the arrangement of the metal-capped colloidal spheres. Upon stretching, the lattice of metal-capped colloidal spheres will be deformed, leading to a large wavelength shift of surface plasmon resonances and simultaneously an obvious color change. This stretchable plasmonic structure offers a promising approach to tune surface plasmon resonances and might be exploited in realizing flexible plasmonic devices with tunability of mechanical strain.
\end{abstract}

\section{KEYWORDS}

Plasmonic structure, metal-capped colloidal spheres, elastomeric substrate, tunable surface plasmon resonance

\section{Introduction}

Plasmonic structures have received increasing attention owing to their unique optical response resulting from the excitations of surface plasmons [1-3]. Surface plasmons are surface charge density oscillations bound at metallic surfaces [4]. The concentration of their optical near-fields and subwavelength nature can render important applications in many fields from bio-sensing to nanophotonics [3].

The optical response of conventional plasmonic structures composed of either metallic nanoparticles or nanostructured continuous metallic films is determined by their structural parameters and remains unalterable once fabricated. To obtain more degrees of freedom in tuning, plasmonic structures which are tunable by external means are highly desirable. The tunability of plasmonic structures can significantly expand their application scope. Up to now, several tuning mechanisms have been proposed, including electrical [5], thermal [6], ferroelectric [7], and mechanical [8] tuning. Except for mechanical tuning, other tuning mechanisms offer a rather small spectral range of tuning. Mechanically tunable plasmonic structures which have already been demonstrated include metal nanoparticles embedded in a polymer matrix [9], and metal gratings $[8,10]$ and nanovoids [11] supported by elastomers. Metal

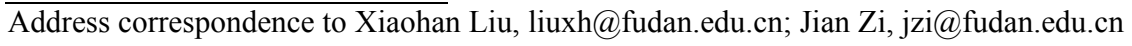


nanoparticles support only localized surface plasmons, while metal gratings support delocalized surface plasmons, but for a single polarization. On the other hand, tunable metal nanovoids [11] can support localized or delocalized surface plasmons depending on their truncations. Their reported spectral tuning range is, however, rather small [11]. Moreover, both tunable metal gratings and nanovoids cannot sustain large strain since fractures occur under these conditions.

In this paper, we describe a simple and inexpensive method, based on self-assembly and transfer-printing techniques [12], to fabricate a highly stretchable plasmonic structure-a monolayer array of metalcapped colloidal spheres on an elastomer substrate. This structure supports delocalized surface plasmons and, under stretching, the surface plasmon resonances undergo a large wavelength shift. This mechanically tunable plasmonic structure can sustain large strain without noticeable fractures in the substrate, or lattice disorder in the metal-capped colloidal spheres.

\section{Experimental}

The procedure for fabrication of the stretchable plasmonic structure is shown schematically in Fig. 1(a). The first step is to prepare a gold-coated quartz slide covered with a monolayer array of polystyrene (PS) spheres. The detailed process is as follows. A 10-nm-thick $\mathrm{Cr}$ cohesive layer was first deposited onto the 1-mm-thick quartz substrate. A 200-nm-thick $\mathrm{Au}$ layer was then deposited on the $\mathrm{Cr}$ layer by sputtering. A monolayer array of monodisperse PS spheres of diameter $600 \mathrm{~nm}$ (size dispersion 1\%) was formed on the Au surface by a self-assembly method [13]. It should be noted that metal surfaces coated with a monolayer array of colloidal spheres show interesting optical responses [14] and can serve as an affinity sensor with high sensitivity [15].

The second step is to prepare an elastomeric substrate. A 1-mm-thick transparent polydimethylsiloxane (PDMS) (Sylgard 184, Dow Corning; the mixing ratio between the polymer precursor and the

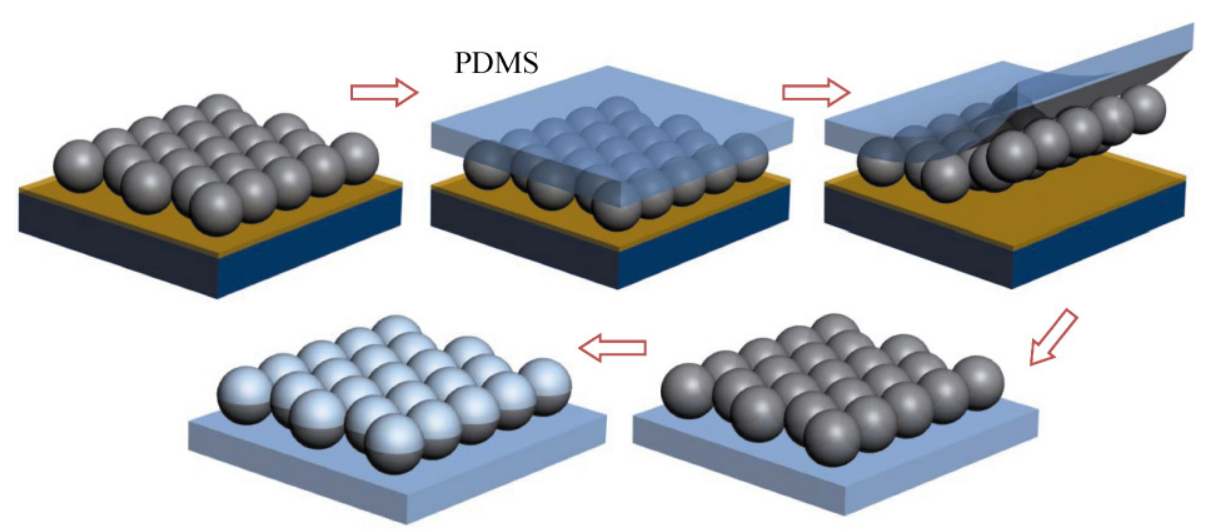

(a)

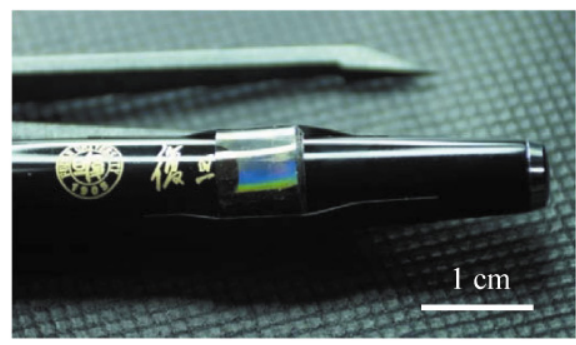

(b)

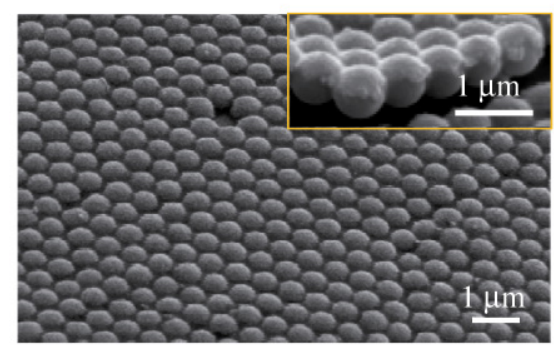

(c)

Figure 1 (a) Sample fabrication procedure. (b) Optical image of a typical sample placed on the round surface of a pen body. (c) SEM image of the sample. The inset shows a close-up image of the metal-capped PS spheres. The metal caps can be clearly seen 
curing agent was 10:1.0) layer was spread on another quartz slide and left to cure at $50{ }^{\circ} \mathrm{C}$ for $12 \mathrm{~h}$ [16]. After curing, the PDMS membrane was carefully peeled off the slide. The third step is to transfer the monolayer array of PS spheres onto the PDMS substrate. To do this, the PDMS film was pasted onto the monolayer array of PS spheres prepared in the first step. The PDMS film was then carefully peeled off, such that the monolayer array of PS spheres was completely transferred onto the PDMS substratethis is due to the large static force of the neat PDMS surface (like adhesive tape) and the relatively much weaker interactions between the colloidal spheres and the Au surface.

The final step is to deposit a 40-nm-thick Ag film on the monolayer array of PS spheres by thermal evaporation. Thus, a monolayer of Ag-capped PS spheres was formed on the elastic PDMS substrate.

Fabricated samples were imaged with a digital camera (Canon EOS 5D). Optical micrographs of the samples under stretching were observed with a Leica microscope (DM6000 M) which was connected to a charge coupled device (CCD) camera. The morphology of the samples was characterized by scanning electron microscopy (SEM) (JEOL FESEM JSM-6701F). Reflection spectra at different incident angles were measured with our homemade macro-optical spectrometer. Microscopic reflection spectra of the samples under stretching were measured with an imaging spectrometer (HORIBA Jobin-Yvon iHR320) which was connected to the Leica microscope.

\section{Results and discussion}

The samples show opalescence due to both diffraction by the array of Ag-capped PS spheres and absorption by surface plasmon resonances (to be discussed later) (Fig. 1(b)). Structural characterization by SEM revealed that the Ag-capped PS spheres are arranged regularly in a triangular lattice (Fig. 1(c)). The Ag caps are almost half-shells. It is worth mentioning that metallic half-shells possess many interesting optical properties [17-19].

The reflection spectra of the fabricated structure were obtained by macro-optical spectroscopy at different incident angles, as shown in Fig. 2. At normal incidence, two prominent reflection dips can be found. With increasing incident angles, the lowwavelength reflection dip is still observed and shifts to higher wavelength. The high-wavelength dip also shifts with incident angle, but almost disappears at large incident angles.

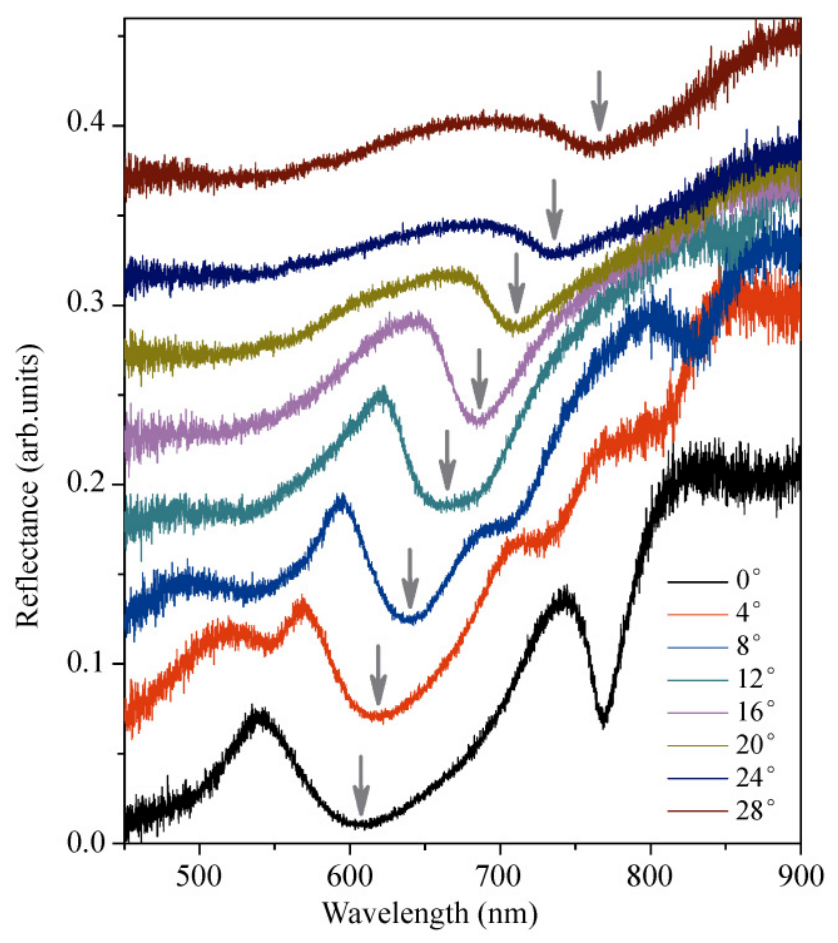

Figure 2 Reflection spectra of the stretchable plasmonic structure at different incident angles measured by macro-optical spectroscopy. Arrows indicate reflection dips corresponding to the resonances of coupled surface plasmons

The existence of reflection dips can be understood in terms of excitations of delocalized surface plasmons, i.e., coupled surface plasmons [1-3]. It is well known that an isolated $\mathrm{Ag}$ cap supports localized surface plasmons. When Ag-capped PS spheres are put together to form a regular two-dimensional (2-D) lattice, localized surface plasmons between neighboring Ag caps can couple with each other provided that their distance is not too large. Due to the periodicity of the 2-D lattice, this coupling leads to a welldefined dispersion of surface plasmons or band structure $\omega\left(k_{\mathrm{SP}}\right)$, where $k_{\mathrm{SP}}$ is the wave-vector of the coupled surface plasmons. If the in-plane wavevector of incident light $k_{\|}$satisfies the following phase-matching condition [1-3]

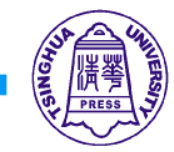

黑 Springer 


$$
\boldsymbol{k}_{\mathrm{SP}}=\boldsymbol{k}_{\|}+\boldsymbol{G}
$$

then coupled surface plasmons can be excited, where $\mathrm{G}$ represents the reciprocal lattice vectors of the 2-D lattice. Therefore, the coupling of incident light with coupled surface plasmons is responsible for the observed reflection dips [20]. The dependence of the reflection dips on incident angles can be understood by the fact that incident light with different incident angles will excite different coupled surface plasmons.

To demonstrate the tunability of the our stretchable plasmonic structure, we observed its color change and also recorded its reflection spectra with a 10x optical microscope when stretching the PDMS substrate, as shown in Fig. 3. Without stretching, the sample appears bright green. With increasing strain, there is an obvious color change from green to brown to dark red at large strain (Figs. 3(a)-3(f)). After being released from the stretched state, the original bright green color was quickly recovered, confirming the reversibility of tuning. The measured reflection spectra confirm these observations. The reflection dip due to the coupled surface plasmons undergoes a wavelength shift towards larger wavelengths with increasing strain (Fig. 3(g)). Figure 3(h) shows that there is an almost linear variation in the reflection dip as a function of strain. The wavelength shift of the reflection dip on stretching is large: at $37.6 \%$ strain, for instance, the wavelength shift is as large as $47 \mathrm{~nm}$. This indicates that the stretchable plasmonic structure has a high tunability.

The triangular lattice of the monolayer array of Ag-capped PS spheres shows a deformation under stretching. Under a strain of about $41 \%$ the triangular lattice of Ag-capped PS spheres is deformed into an almost square lattice (Fig. 4(b)), which is also confirmed
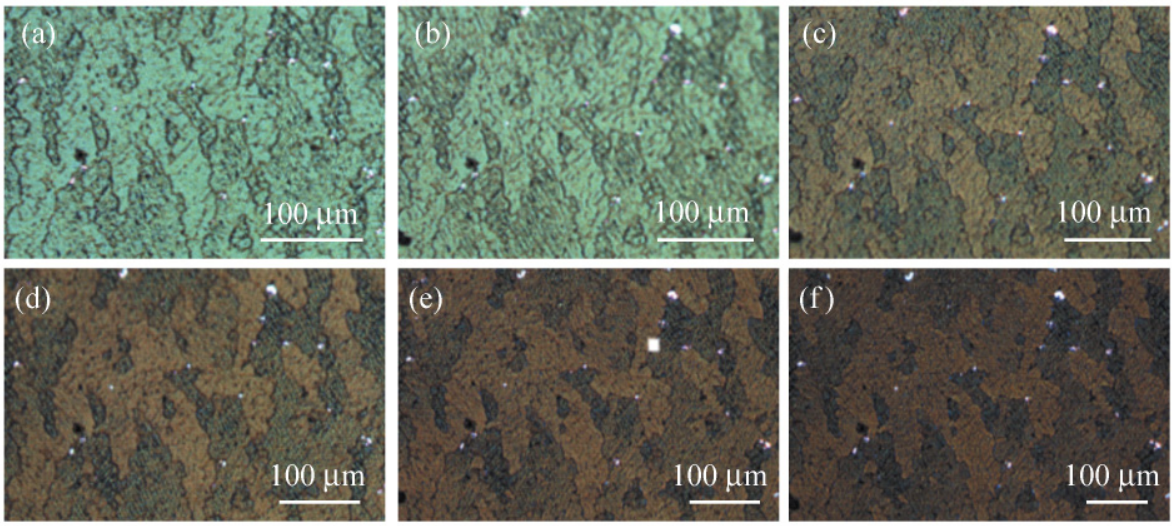

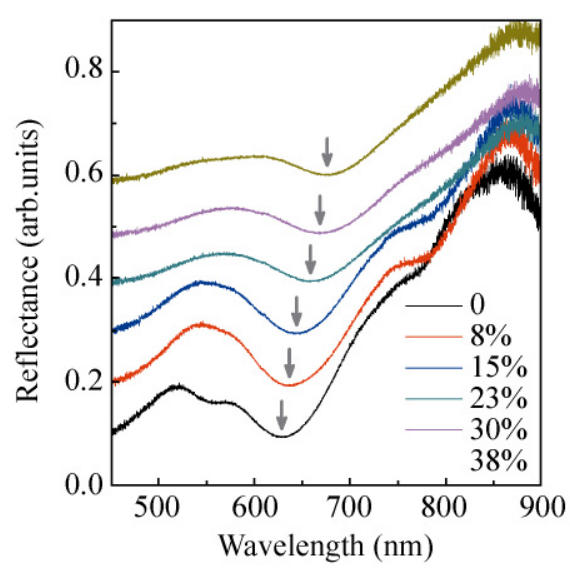

(g)

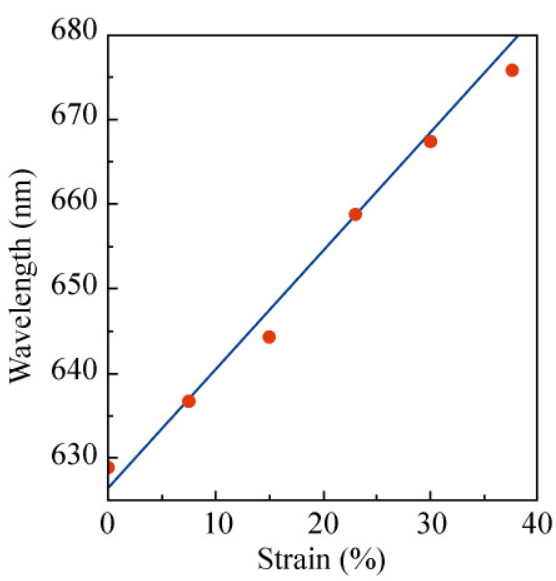

(h)

Figure 3 Colors of the sample and reflection spectra measured by micro-optical spectroscopy under different applied strain: (a) 0 , (b) $8 \%$, (c) $15 \%$, (d) $23 \%$, (e) $30 \%$, and (f) $38 \%$ strain. (g) Spectra of the samples in (a)-(f) are shown from bottom to top, where the arrows indicate the surface plasmon resonances. (h) Wavelengths of the surface plasmon resonance as a function of strain. The dots and the line represent measured and fitted results, respectively 

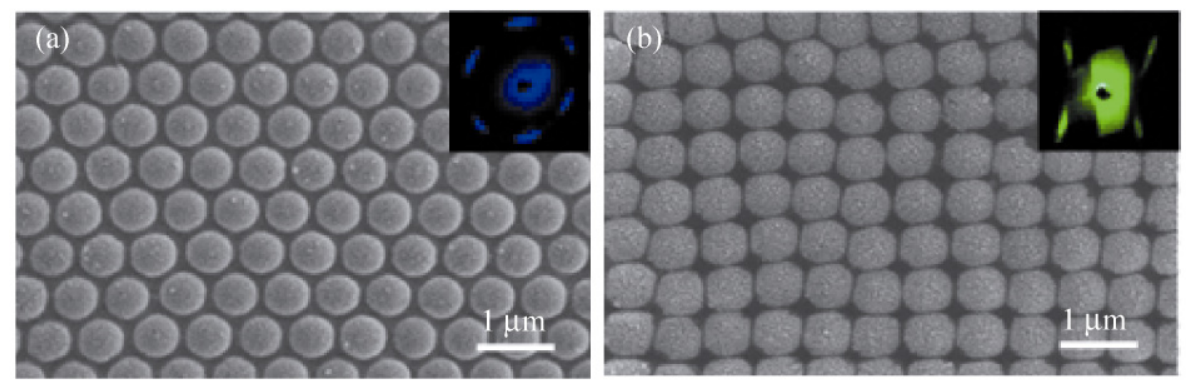

Figure 4 SEM images of Ag-capped PS spheres on the PDMS substrate with (a) zero and (b) $41 \%$ strain applied. Insets show the light diffraction patterns

by the light diffraction pattern. Note that the coupled surface plasmons supported by the stretchable plasmonic structure depend strongly on the coupling of surface plasmons between neighboring Ag caps. As a result, their wavelengths depend not only on the lattice type, but also on the distance between neighboring Ag caps. Without any applied strain, the lattice is triangular with a coordination number of six. The coupling of surface plasmons between neighboring Ag caps is a maximum. Under strain, the lattice becomes deformed and the coordination number decreases below six. This will lead to a decrease in the coupling of surface plasmons between neighboring Ag caps, eventually resulting in the observed upward wavelength shift of the reflection dips with increasing strain.

Our plasmonic structure is highly stretchable. After releasing from stretching under more than 50\% strain, the original bright green color of the structure is restored. SEM images also revealed that, even under more than $50 \%$ strain, there are neither fractures in the PDMS substrate nor disorder in the lattice of Ag-capped PS spheres.

\section{Conclusions}

A highly stretchable plasmonic structure consisting of a monolayer array of Ag-capped PS spheres on an elastic PDMS substrate has been fabricated using self-assembly and transfer-printing techniques. The structure shows surface plasmon resonances which can be tuned by stretching, leading to a reversible color change. The large shift in surface plasmon resonances and color change under strain suggest that this tunable plasmonic structure might find niche applications from sensing to display.

\section{Acknowledgements}

This work was supported by the National Program on Key Basic Research Project (973 Program) (Grant Nos. 2007CB613200 and 2006CB921700). The research of J. Z. and X. H. L. is further supported by the National Natural Science Foundation of China (NSFC) and the Shanghai Science and Technology Commission.

Open Access: This article is distributed under the terms of the Creative Commons Attribution Noncommercial License which permits any noncommercial use, distribution, and reproduction in any medium, provided the original author(s) and source are credited.

\section{References}

[1] Barnes, W. L.; Dereux, A.; Ebbesen, T. W. Surface plasmon subwavelength optics. Nature 2003, 424, 824-830.

[2] Zayats, A. V.; Smolyaninov, I. I.; Maradudin, A. A. Nanooptics of surface plasmon polaritons. Phys. Rep. 2005, 408, 131-314.

[3] Maier, S. A. Plasmonics: Fundamentals and Applications; Springer: New York, 2007.

[4] Raether, H. Surface Plasmons; Springer: Berlin, 1988.

[5] Dickson, W.; Wurtz, G. A.; Evans, P. R.; Pollard, R. J.; Zayats, A. V. Electronically controlled surface plasmon dispersion and optical transmission through metallic hole arrays using liquid crystal. Nano Lett. 2008, 8, 281-286.

[6] Xu, G.; Huang, C. M.; Tazawa, M.; Jin, P.; Chen, D. M. 
Nano-Ag on vanadium dioxide. II. Thermal tuning of surface plasmon resonance. J. Appl. Phys. 2008, 104, 053102.

[7] Chen, H. L.; Hsieh, K. C.; Lin, C. H.; Chen, S. H. Using direct nanoimprinting of ferroelectric films to prepare devices exhibiting bi-directionally tunable surface plasmon resonances. Nanotechnology 2008, 19, 435304.

[8] Rehwald, S.; Berndt, M.; Katzenberg, F.; Schwieger, S.; Runge, E.; Schierbaum, K.; Zerulla, D. Tunable nanowires: An additional degree of freedom in plasmonics. Phys. Rev. B 2007, 76, 085420 .

[9] Chiang, Y. L.; Chen, C. W.; Wang, C. H.; Hsieh, C. Y.; Chen, Y. T.; Shih, H. Y.; Chen, Y. F. Mechanically tunable surface plasmon resonance based on gold nanoparticles and elastic membrane polydimethylsiloxane composite. Appl. Phys. Lett. 2010, 96, 041904.

[10] Olcum, S.; Kocabas, A.; Ertas, G.; Atalar, A.; Aydinli, A. Tunable surface plasmon resonance on an elastomeric substrate. Opt. Express 2009, 17, 8542-8547.

[11] Cole, R. M.; Mahajan, S.; Baumberg, J. J. Stretchable metal-elastomer nanovoids for tunable plasmons. Appl. Phys. Lett. 2009, 95, 154103.

[12] Packard, C. E.; Murarka, A.; Lam, E. W.; Schmidt, M. A.; Bulović, V. Contact-printed microelectromechanical systems. Adv. Mater. 2010, 22, 1840-1844.

[13] Gates, B.; Qin, D.; Xia, Y. N. Assembly of nanoparticles into opaline structures over large areas. Adv. Mater. 1999, $11,466-469$.
[14] Shi, L.; Liu, X. H.; Yin, H. W.; Zi, J. Optical response of a flat metallic surface coated with a monolayer array of latex spheres. Phys. Lett. A 2010, 374, 1059-1062.

[15] Yu, X. D.; Shi, L.; Han. D. Z.; Zi, J.; Braun, P. V. High quality factor metallodielectric hybrid plasmonic-photonic crystals. Adv. Funct. Mater. 2010, 20, 1910-1916.

[16] Zhou, Y. X.; Hu, L. B.; Grüner, G. A method of printing carbon nanotube thin films. Appl. Phys. Lett. 2006, 88, 123109.

[17] Love, J. C.; Gates, B. D.; Wolfe, D. B.; Paul, K. E.; Whitesides, G. M. Fabrication and wetting properties of metallic half-shells with submicron diameters. Nano Lett. 2002, 2, 891-894.

[18] Zhan, P.; Wang, Z. L.; Dong, H.; Sun, J.; Wu, J.; Wang, H. T.; Zhu, S. N.; Ming, N. B.; Zi, J. The anomalous infrared transmission of gold films on two-dimensional colloidal crystals. Adv. Mater. 2006, 18, 1612-1616.

[19] Li, Y. Y.; Pan, J.; Zhan, P.; Zhu, S. N.; Ming, N. B.; Wang, Z. L.; Han, W. D.; Jiang, X. Y.; Zi, J. Surface plasmon coupling enhanced dielectric environment sensitivity in a quasi-three-dimensional metallic nanohole array. Opt. Express 2010, 18, 3546-3555.

[20] Liu, Z.; Shi, L.; Shi, Z.; Liu, X. H.; Zi, J.; Zhou, S. M.; Wei, S. J.; Li, J.; Zhang, X.; Xia, Y. J. Magneto-optical Kerr effect in perpendicularly magnetized $\mathrm{Co} / \mathrm{Pt}$ films on twodimensional colloidal crystals. Appl. Phys. Lett. 2009, 95, 032502 . 